\title{
Trauma and COVID-19 infection
}

\section{Jerzy Lasek, Piotr Brzeziński}

Department of Physiotherapy and Medical Emergency, Institute of Health Sciences, Pomeranian University of Stupsk, Poland

Corresponding author: Prof. Jerzy Lasek, MD PhD, E-mail: jerzy.lasek@apsl.edu.pl

Sir,

COVID-19 pandemic which started in 2019 dramatically influenced healthcare systems all over the world. This epidemic affected both epidemiology and organization of management in patients after severe trauma. In many countries emergency departments suddenly were put under pressure by an overwhelming number of patients with COVID-19 infection and with acute respiratory insufficiency requiring respirator therapy in intensive care units (ICU). In many countries special guidelines for COVID-19 management have been established. In general the aim of the guidelines is to preserve the hospital capacity for the patients following both major and minor trauma by means of reorganization of local health systems and providing optimal trauma care [1].

Many authors found that an absolute number of trauma patients admitted to emergency facilities was reduced, mainly due to lower number of road traffic accidents. In the Chinese reports a reduction of trauma patients by $39 \%$ with a deep decrease in the number of male patients. Pre - hospital mortality was unexpectedly higher with an increase of intentional injuries from interpersonal violence [2-4]. A reduction in orthopaedic admissions was reported in Europe. Staying home during COVID-19 pandemic decreased number of fractures in adults and children. The mean age of the patients was significantly higher which itself imposed higher demands to the trauma services. The severity of injuries measured by Injury Severity Score (ISS) in many units was similar to that preceding the pandemic and fewer patients with major trauma were admitted to the surgical departments in some European studies. But in the American studies the ISS was higher. It has also been been concluded that the stress induced by the COVID-19 produces higher risk of dangerous behaviour of some persons. Traffic related trauma decreased, but falls occurring in domestic settings were more frequent. No difference was observed in the anatomical distribution of injuries in the region of head, thorax, abdomen and skeletal system. In most reports the incidence of emergency surgical procedures performed within 24 hours was similar with that recorded in the pre - COVID period [5]. In clinical observations the virus pandemic highly increased the risk of serious complications and death among traumatized patients from road traffic accidents and assault following gunshot wounds and stabbings. It has been found that infected patients in trauma centers had six times higher risk of death than patients with similar injuries without this virus. COVID-19 positive patients demonstrate two times higher likelihood of severe complications such as pulmonary insufficiency, tracheal intubation, thromboembolism, neurological deficits, cardiac and renal failure [6]. It must be remembered that COVID-19 also imposes extremely strong impact on patients with minor injuries resulting worse outcome in those who would have otherwise expected to do well. The prognosis depends on the combination of trauma and COVID-19 pathological cascade which is not fully understood yet. By all means it is recommended that minor injuries should never be underestimated in traumatized patients with COVID-19. Various pandemic recommendations should never block elective operations of these patients although some delay in COVID-19 infected patients may be necessary.

During COVID-19 pandemic a big challenge is to maintain an adequate level of care in facilities for other pathological states such as strokes, cardiac attacks (STEMI) and other emergencies. Some authors complain of decreased cancer screenings resulting delayed oncological diagnosis and management. This complain should be seriously taken into consideration by healthcare organizers. It is imperative that both

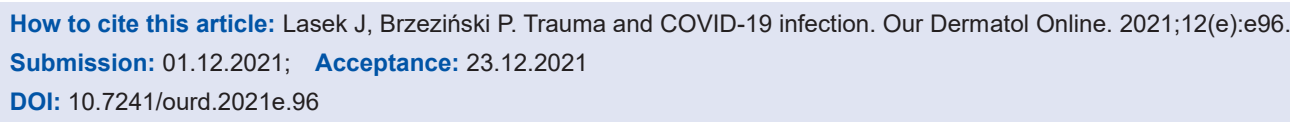


emergency and elective surgery for the patients with COVID-19 following severe and minor trauma should be performed by experienced and perfectly prepared medical personnel in well equipped surgical facilities.

\section{REFERENCES}

1. Giudici R, Lancioni A, Gay H. Impact of COVID-19 on severe trauma trends and healthcare system reassessment in Lombardia Italy: an analysis from the regional trauma registry. World Emerg. Surg. 2021;16:39.

2. Yang F, Lu X. The effect of COVID-19 on trauma system in one city of China. Scand J Trauma Resusc Emerg Med. 2020;28:57.

3. Sigha OB, Kouotou EA. [COVID-19 infection revealed by a flare-up of psoriasis in an elderly Cameroonian: about a case]. Our Dermatol
Online. 2021;12(Supp. 1):16-20.

4. Petrov A, Vasileva M. Effect of platelet-rich plasma on diffuse effluvium in post-COVID-19 infection. Our Dermatol Online. 2021;12:267-9.

5. Ohliger E, Umpierrez E, Buehler L, Ohliger AW, Magister S, Vallier $\mathrm{H}$, et al. Mental health of orthopaedic trauma patients during the 2020 COVID-19 pandemic. Int Orthop. 2020;44:1921-5.

6. Kaufman EJ, Ong AW, Cipolle MD, Whitehorn G, Ratnasekera A, Stawicki SP, et al. The impact of COVID-19 infection on outcomes after injury in a state trauma system. J Trauma Acute Care Surg. 2021;91:559-65.

Copyright by Jerzy Lasek, et al. This is an open-access article distributed under the terms of the Creative Commons Attribution License, which permits unrestricted use, distribution, and reproduction in any medium, provided the original author and source are credited.

Source of Support: Nil, Conflict of Interest: None declared. 